\title{
Immunologic strategies and outcomes in ABO- incompatible living donor liver transplantation
}

\author{
Jongwook $\mathrm{Oh}^{1}$ and Jong Man $\mathrm{Kim}^{2}$ \\ ${ }^{1}$ Department of Surgery, Samsung Changwon Hospital, Sungkyunkwan University School of Medicine, Changwon; ${ }^{2}$ Department of \\ Surgery, Samsung Medical Center, Sungkyunkwan University School of Medicine, Seoul, Korea
}

Antibody mediated rejection (AMR) after adult ABO-incompatible living donor liver transplantation (ABO-I LDLT) induced hepatic necrosis or diffuse intrahepatic biliary complications, which were related with poor graft and patient survival. Various desensitization protocols have been used to overcome these problems. Since using rituximab, the outcomes of ABO-I LDLT show a similar survival rate to those of ABO-compatible living donor liver transplantation. However, diffuse bile duct complications still occur after ABO-I LDLT. We have reviewed the past and current immune strategies for desensitization and to provide outcomes and ABO incompatibility-related complications in ABO-I LDLT. (Clin Mol Hepatol 2020;26:1-6)

Keywords: Immunosuppression; Rejection; Graft survival; Complications

\section{INTRODUCTION}

In the past, adult $\mathrm{ABO}$ incompatible living donor liver transplantation (ABO-I LDLT) had poor graft survival and low patient survival due to hyperacute rejection and a high risk of vascular biliary complication, and it was considered a contraindication. ${ }^{1-4}$ Susceptibility to rejection, including severe hepatic necrosis and diffuse intravascular coagulation disorder within the graft, appears to be due to the blood group antigen expressed in the vascular endothelium and bile ducts after transplantation. ${ }^{5,6}$ Various desensitization strategies have been introduced to overcome the barrier of $\mathrm{ABO}$ incompatibility. ${ }^{7-9}$ However, desensitization protocols differ at each center, and the necessity of local infusion, splenectomy, intravenous immunoglobulin (IVIG), and plasmapheresis is contro- versial. After the rituximab era, the outcome of ABO-I LDLT has been reported in many studies to be comparable to $\mathrm{ABO}$ compatible living donor liver transplantation (ABO-C LDLT). ${ }^{10-13}$ Many centers are now trying to simplify protocols. ${ }^{14-20}$ We review the past and current immune strategies for desensitization and to provide outcomes and $A B O$ incompatibility-related complications in ABO-I LDLT.

\section{HISTORY}

Thomas Starzl introduced liver transplantation (LT) for the ABO blood group in 1969. In addition, Thomas Starzl proposed the "liver is privileged organ" concept since the liver in contrast to

\footnotetext{
Abbreviations:

ABO-C LDLT, ABO compatible living donor liver transplantation; ABO-I LDLT, $A B O$ incompatible living donor liver transplantation; $A M R$, antibody mediated rejection; DIC, disseminated intravascular coagulation; DIHC, diffuse intrahepatic biliary complications; HCC, hepatocellular carcinoma; IgG, immunoglobulin G; $\lg \mathrm{M}$, immunoglobulin $\mathrm{M}$; IVIG, intravenous immunoglobulin
}

\section{Corresponding author : Jong Man Kim}

Department of Surgery, Samsung Medical Center, Sungkyunkwan University School of Medicine, 81 Irwon-ro, Gangnam-gu, Seoul 06351, Korea

Tel: +82-2-3410-1719, Fax: +82-2-3410-0040

E-mail:yjongman21@gmail.com

https://orcid.org/0000-0002-1903-8354

Received : Feb. 10, 2019 / Accepted : Feb. 18, 2019

Copyright $\odot 2020$ by Korean Association for the Study of the Liver

This is an Open Access article distributed under the terms of the Creative Commons Attribution Non-Commercial License (http://creativecommons.org/licenses/by-nc/3.0/) which permits unrestricted non-commercial use, distribution, and reproduction in any medium, provided the original work is properly cited. 
heart or kidney transplantations, resists acute rejection in animal experimental studies. ${ }^{9}$ Therefore, Starzl's group only reported 11 ABO-I pediatric LT cases in 1979 because of the difficulty of finding compatible small grafts. However, they had shown no evidence of acute rejection in those patients. ${ }^{21}$

In the 1980s and in the early 1990s, ABO incompatible liver transplantation (ABO-I LT) had extremely poor surgical outcomes; severe rejection, hepatic artery thrombosis and intrahepatic bile duct injury were common. Demetris et al. reported a pathological feature, 'single organ disseminated intravascular coagulation (DIC)' in a failed ABO-I liver graft. 'In a control matched study that included 15 ABO-I LT, Sanchez-Urdazpal et al. confirmed increased incidence of cholangitis, bile leak, cellular rejection, and hepatic artery thrombosis in the ABO-I group. ${ }^{22}$ To overcome ABO-incompatibility complications, high dose immunosuppression, splenectomy and plasmapheresis were implemented, but these had little effect on the poor outcomes and increased the occurrence of complications, including infection and sepsis. ${ }^{2,3}$

\section{DESENSITIZATION MANAGEMENT}

\section{Plasmapheresis or total plasma exchange}

Anti-ABO antibodies are thought to cause antibody mediated rejection (AMR) in ABO-I LT. It has been reported that hepatic necrosis and intrahepatic biliary complications in ABO-I LDLT are closely related to high perioperative anti-A or anti-B antibody titers. ${ }^{6}$ Plasmapheresis has been reported as rapidly reducing antiblood type isoagglutinin titers for ABO-I LDLT. ${ }^{23}$ Therefore, plasmapheresis has been applied prior to LT in order to reduce antiblood antibodies to levels considered safe enough to improve the outcomes of ABO-I LDLT. However, it has also been reported that even if antibody titers are reduced by plasmapheresis prior to ABO-I LDLT, isoagglutinin titers can rise again within 3-7 days after operation. Although plasmapheresis is able to remove antibodies from the peripheral blood prior to ABO-I LT, plasmapheresis cannot suppress the production of new antibodies from the preexisting plasma cells. For this reason, repetitive plasmapheresis has been considered an efficient therapeutic method in patients with a rise in isoagglutinin titers after ABO-I LDLT. ${ }^{16}$ It has been previously reported that the target of pre-transplant antibody ABO-titer values following plasmapheresis were less than 1:8, $1: 16,1: 32$ or $1: 64$ to prevent posttransplant $A M R{ }^{14}$ The target titer differs markedly by center and a standard target titer has not yet been established.

\section{Splenectomy}

Splenectomy has been an important part of the protocol for ABO-I LDLT at many centers because the spleen is the body's major antibody producing organ, and contains large amounts of $B$ cells and plasma cells. It also fulfils particular functions in blood filtration, phagocytosis, erythrocyte destruction, antigen uptake and potential hemopoiesis. Splenectomy in ABO-I LDLT carries risks for severe post-transplant infection and portal vein thrombosis, pancreatic fistula, and sepsis. ${ }^{20}$ In addition, splenectomy is time-consuming and can cause massive bleeding as a result of splenomegaly in patients with severe liver cirrhosis. ${ }^{19}$ However, several studies have reported that splenectomy had not decreased the incidence of AMR. ${ }^{13,19}$ Raut et al. had found no statistically significant difference in anti-ABO immunoglobulin $\mathrm{M}(\operatorname{IgM})$ and immunoglobulin $\mathrm{G}$ ( $\mathrm{lgG}$ ) antibody titers between splenectomy and non-splenectomy groups. ${ }^{20}$ Therefore, the omission of splenectomy in ABO-I LDLT has recently emerged with the prophylactic use of rituximab.

\section{IVIG}

The mechanism of action of IVIG is complex and not completely understood. It has been proposed to include the blocking of Fc receptors to non-nuclear cells, direct antibody neutralization, suppression of CD19 presentation to activated B cells, suppression of complements, and suppression of all porous T cells. ${ }^{17-19}$ Several previous studies have reported on the effectiveness of additional IVIG for preventing AMR. Kim et al. had reported that the combination of rituximab, plasmapheresis, and IVIG had excellent results. ${ }^{15}$ However, a Japanese study had reported that AMR incidence does not significantly differ between desensitization regimens with or without IVIG. ${ }^{24}$

\section{Local graft infusion therapy}

Local infusion therapy was reported in 1998, and involved methylprednisolone, prostaglandin E1, and gabexate mesilate administered via a catheter through the portal vein. ${ }^{25}$ This regimen increased survival from $22 \%$ to $60 \%{ }^{26}$ The theoretical basis of these local infusion agents is that they inhibit different key reactions in single-organ disseminated intravascular coagulation triggered by preformed antibodies against the donor antigen. Prosta- 
glandin E1 improves microcirculation through vasodilatation and the prevention of platelet thrombi. ${ }^{27}$ Gabexate mesilate is a protease inhibitor that inhibits platelet aggregation and coagulation factors. ${ }^{26}$ Meanwhile, hepatic artery infusion therapy was introduced to avoid portal vein thrombosis by portal vein infusion therapy ${ }^{28}$ However, catheter-related complications, including vascular thrombosis, infection, bleeding, and dislocation, have been reported in $37 \%$ of patients undergoing portal vein infusion therapy, $22 \%$ of patients undergoing portal vein and hepatic artery infusion therapy, and $16 \%$ of patients underoing hepatic artery infusion therapy. ${ }^{8}$ These complications can be life-threatening. Kim et al. showed no difference in liver function tests between patients undergoing local infusion therapy and systemic infusion, respectively. ${ }^{10}$ Song et al. also reported that there were no significant differences in the incidence of AMR and patient survival between a "Local infusion group" and a "non-Local infusion group" after maintaining the pre-transplant $A B O$ antibody titer within a limited range using plasmapheresis. ${ }^{13}$

\section{Rituximab}

Rituximab is a monoclonal chimeric human-murine anti-CD20 antibody that depletes $B$ cells by complement-dependent cellular cytotoxicity. ${ }^{8,29}$ It depletes CD20-positive B cells from circulation and lymphoid tissues including the spleen. ${ }^{8,29}$ Thus, rituximab acts as a form of chemical splenectomy. Several previous studies have shown that rituximab's effect on B cells in peripheral blood lasts for several months, removing cells within 48-72 hours. ${ }^{24,29,30}$ Moreover, most data show that a single dose of rituximab is sufficient for suppressing $B$ cells in the peripheral blood. The number of $B$ cells in peripheral blood decreased in three days after a single dose of rituximab $\left(375 \mathrm{mg} / \mathrm{m}^{2}\right)$, and the number of cells in the peripheral blood were completely eliminated after more than three weeks. ${ }^{31}$ Regular and multiple rituximab doses increased the incidence of fungi and cytomegalovirus infections. ${ }^{13}$ Therefore, repeated administration of rituximab may be unnecessary and may increase the risk of serious infection due to long-term hypoglycemia. Currently, most centers administer a single dose of rituximab (300 or $375 \mathrm{mg} / \mathrm{m}^{2}$ ), two weeks before surgery.

When the outcomes of ABO-I LDLT are divided into before and after the rituximab era, a Japanese nationwide survey revealed that the 3-year survival rates increased from $30 \%$ to $80 \%$ after the introduction of rituximab. ${ }^{8}$ In a study that included 381 adult patients in the Japanese registry of ABO-I LDLT, only the absence of rituximab prophylaxis was a significant risk factor for AMR.
Thus, rituximab prophylaxis significantly decreased the incidence of AMR. In that study, the incidence of AMR decreased from $23.5 \%$ to $6.2 \%$ after the introduction of rituximab. ${ }^{24}$ Since the rituximab era, many centers are now trying to simplify the protocol, and avoid splenectomy, local graft infusion, IVIG, and plasmapheresis. ${ }^{14-20}$

\section{Desensitization trials without plasmapheresis}

The role of preoperative $A B O$ antibody titer in rejection of $A B O-I$ LDLT has not yet been established. Several studies have shown no significant correlation between $\mathrm{ABO}$ antibody titer and AMR, indicating that high preoperative antibody values have no significant effect on AMR frequency. ${ }^{8,32}$ Another study suggested that sufficient desensitization could be achieved using rituximab alone. $^{16}$ In addition, another study included a simplified protocol using rituximab and IVIG without plasmapheresis for ABO-I LDLT. The study reported that the protocol was safe and effective in achieving sufficient desensitization and showed comparable outcomes in patients with titers no higher than 1:64. ${ }^{15}$ The incidence of diffuse intrahepatic biliary complications (DIHC) was 3-5\%. . $^{11-13}$ Considering intraoperative blood loss was much during $\mathrm{ABO}-\mathrm{I}$ LDLT and very low incidence of DIHC, desensitization protocol without preoperative plasmapheresis required more search for validation.

\section{OUTCOMES}

Hyperacute rejection has not been reported in most studies since the use of rituximab. Kim et al. reported 100\% patient and graft survivals and no AMR in $22 \mathrm{ABO}-\mathrm{I}$ LDLT patients with titers adjusted below 1:32 by total plasma exchange. ${ }^{10}$ They reported five biliary complication cases. Song et al. also reported that patient survival, graft survival, and biopsy proven acute rejection were not significantly different between ABO-I LDLT and ABO-C LDLT. ${ }^{11}$ They showed that diffuse intrahepatic bile duct complications were observed in 12 cases in the ABO-I LDLT group. A recent study showed that 47 ABO-I LDLT patients who were compared to a 1:2 matched 94 ABO-C LDLT group did not show significant differences in survival and acute rejection, as well as biliary complications. ${ }^{12}$ However, three DIHC cases occurred in the ABO-I LDLT patients and progressed to graft failure.

It is not known whether rituximab prophylaxis for desensitization affects hepatocellular carcinoma (HCC) recurrence in ABO-I 
LDLT. Kim et al. ${ }^{33}$ reported that ABO incompatibility was not associated with $\mathrm{HCC}$ recurrence. The 1-, 2-, and 3-year disease-free survival rates in ABO-I LDLT and ABO-C LDLT groups were 90.3\%, $79.7 \%$, and $73.3 \%$ and $86.7 \%, 79.0 \%$, and $75.3 \%$, respectively $(P=0.96) .^{33}$ The overall patient survival rates for the same period in the ABO-I LDLT and ABO-C LDLT groups were 90.6\%, 85.0\%, and $81.9 \%$ and $88.0 \%, 83.5 \%$, and $82.5 \%$, respectively $(P=0.77){ }^{33}$ They had shown that AFP, tumor size, encapsulation and microcirculation invasion were associated with $\mathrm{HCC}$ recurrence except in ABO-incompatibility. Propensity score match study had shown comparable recurrence-free survival rates and overall patient-survival outcomes between ABO-I LDLT and ABO-C LDLT groups. $^{34}$

\section{COMPLICATIONS}

Egawa et al. reported two types of graft failure in ABO-I LDLT. The first, 'liver necrosis' occurred acutely 1-2 weeks after transplantation, leading to massive graft necrosis within a month. The second, 'intrahepatic bile duct injury' presented more slowly 2-3 months after transplantation, with development of extensive irregularities of the intrahepatic bile duct, resulting in graft failure. These reactions were not observed in children $<1$ year of age, whose ability to produce antibodies against blood group antigens had not yet been established. The 5 -year survival rate of recipients younger than 1 year (infants) and 16 years or older (adults) was reported as $76 \%$ and $22 \%$, respectively. Because of poor survival outcomes and high incidence of complications, ABO-I LDLT became unpopular and was reserved for emergency transplant surgery only. 35,36

Diffuse intrahepatic bile duct complications were significantly higher in ABO-I LDLT than in ABO-C LDLT. Because the targets of isoagglutinin are the bile duct's epithelium and vascular endothelium of the graft, microvascular thrombotic occlusion of graft bile duct can occur, which causes ischemic cholangiopathy. Although the fulminant hepatic necrosis caused by severe AMR in ABO-I LDLT has been overcome since the introduction of rituximab, the risk of attenuated AMR still remains." Attenuated AMR can cause DIHC. Unlike fulminant necrosis, DIHC is not always fatal. However, DIHC eventually leads to refractory cholangitis, which leads to sepsis and graft failure. In most cases, DIHC cannot be treated by conventional biliary interventions. The only proven effective treatment is re-transplantation. In addition, DIHC degrades the quality of patient life due to frequent recurrent episodes of cholangitis and the need for intervention and readmission."

\section{CONCLUSION}

In conclusion, ABO-I LDLT is a very effective and safe method for extending a raw pool of liver donors. Survival outcomes are now comparable with rituximab prophylaxis and plasmapheresis. However, there is still concern about the high incidence of biliary complication especially DIHC, an intractable form of biliary stenosis that can occur regardless of the isoagglutinin titer. Therefore, we need to closely follow the patient course over several months after $A B O-I$ LDLT even in patients with very low isoagglutinin titers after ABO-I LDLT. In the future, we need to identify certain risks and precautions through studies involving immunology and adaptive mechanisms in ABO-I LDLT.

\section{Authors' contributions}

All authors wrote and approved the final version.

\section{Conflicts of Interest}

The authors of this manuscript have no conflicts of interest to disclose.

\section{REFERENCES}

1. Demetris AJ, Jaffe R, Tzakis A, Ramsey G, Todo S, Belle S, et al. Antibody-mediated rejection of human orthotopic liver allografts. A study of liver transplantation across ABO blood group barriers. Am J Pathol 1988;132:489-502.

2. Gugenheim J, Samuel D, Reynes M, Bismuth H. Liver transplantation across ABO blood group barriers. Lancet 1990;336:519-523.

3. Farges 0 , Kalil AN, Samuel D, Saliba F, Arulnaden JL, Debat $P$, et al. The use of $A B O$-incompatible grafts in liver transplantation: a life-saving procedure in highly selected patients. Transplantation 1995;59:1124-1133.

4. Lo CM, Shaked A, Busuttil RW. Risk factors for liver transplantation across the ABO barrier. Transplantation 1994;58:543-547.

5. Kawagishi N, Satomi S. ABO-incompatible living donor liver transplantation: new insights into clinical relevance. Transplantation 2008;85:1523-1525.

6. Egawa H, Oike F, Buhler L, Shapiro AM, Minamiguchi S, Haga $H$, et al. Impact of recipient age on outcome of $\mathrm{ABO}$-incompatible livingdonor liver transplantation. Transplantation 2004;77:403-411.

7. Ikegami T, Taketomi A, Soejima Y, Yoshizumi T, Uchiyama H, Harada 
$\mathrm{N}$, et al. Rituximab, IVIG, and plasma exchange without graft local infusion treatment: a new protocol in $\mathrm{ABO}$ incompatible living donor liver transplantation. Transplantation 2009;88:303-307.

8. Egawa H, Teramukai S, Haga H, Tanabe M, Fukushima M, Shimazu M. Present status of $\mathrm{ABO}$-incompatible living donor liver transplantation in Japan. Hepatology 2008;47:143-152.

9. Raut $V$, Uemoto S. Management of ABO-incompatible livingdonor liver transplantation: past and present trends. Surg Today 2011:41:317-322.

10. Kim JM, Kwon CH, Joh JW, Kang ES, Park JB, Lee JH, et al. ABOincompatible living donor liver transplantation is suitable in patients without ABO-matched donor. J Hepatol 2013;59:1215-1222.

11. Song GW, Lee SG, Hwang S, Kim KH, Ahn CS, Moon DB, et al. Biliary stricture is the only concern in $\mathrm{ABO}$-incompatible adult living donor liver transplantation in the rituximab era. J Hepatol 2014;61:575-582.

12. Kim JM, Kwon CH, Joh JW, Han SB, Sinn DH, Choi GS, et al. Casematched comparison of $\mathrm{ABO}$-incompatible and $\mathrm{ABO}$-compatible living donor liver transplantation. Br J Surg 2016;103:276-283.

13. Song GW, Lee SG, Hwang S, Kim KH, Ahn CS, Moon DB, et al. ABOincompatible adult living donor liver transplantation under the desensitization protocol with rituximab. Am J Transplant 2016;16:157-170.

14. Yamamoto H, Uchida K, Kawabata S, Isono K, Miura K, Hayashida S, et al. Feasibility of monotherapy by rituximab without additional desensitization in $\mathrm{ABO}$-incompatible living-donor liver transplantation. Transplantation 2018;102:97-104.

15. Kim SH, Lee EC, Shim JR, Park SJ. A simplified protocol using rituximab and immunoglobulin for $\mathrm{ABO}$-incompatible low-titre living donor liver transplantation. Liver Int 2018;38:932-939.

16. Lee EC, Kim SH, Shim JR, Park SJ. A comparison of desensitization methods: rituximab with/without plasmapheresis in $\mathrm{ABO}$-incompatible living donor liver transplantation. Hepatobiliary Pancreat Dis Int 2018;17:119-125.

17. Kim JD, Choi DL, Kim SG, Lee AJ. Single-center experience of ABOincompatible living-donor liver transplantation with a new simplified intravenous immunoglobulin protocol: a propensity score-matching analysis. Transplant Proc 2016;48:1134-1138

18. Lee J, Lee JG, Lee JJ, Kim MS, Ju MK, Choi GH, et al. Results of ABOincompatible liver transplantation using a simplified protocol at a single institution. Transplant Proc 2015;47:723-726.

19. Lee SD, Kim SH, Kong SY, Kim YK, Lee SA, Park SJ. ABO-incompatible living donor liver transplantation without graft local infusion and splenectomy. HPB (Oxford) 2014;16:807-813.

20. Raut V, Mori A, Kaido T, Ogura Y, Taku I, Nagai K, et al. Splenectomy does not offer immunological benefits in $\mathrm{ABO}$-incompatible liver transplantation with a preoperative rituximab. Transplantation 2012:93:99-105.

21. Starzl TE, Koep LJ, Halgrimson CG, Hood J, Schroter GP, Porter KA, et al. Fifteen years of clinical liver transplantation. Gastroenterology
1979;77:375-388.

22. Sanchez-Urdazpal L, Sterioff S, Janes C, Schwerman L, Rosen C, Krom RA. Increased bile duct complications in $A B O$ incompatible liver transplant recipients. Transplant Proc 1991;23(1 Pt 2):1440-1441.

23. Kozaki K, Egawa H, Ueda M, Oike F, Yoshizawa A, Fukatsu A, et al. The role of apheresis therapy for $A B O$ incompatible living donor liver transplantation: the Kyoto University experience. Ther Apher Dial 2006;10:441-448.

24. Egawa H, Teramukai S, Haga H, Tanabe M, Mori A, Ikegami T, et al. Impact of rituximab desensitization on blood-type-incompatible adult living donor liver transplantation: a Japanese multicenter study. Am J Transplant 2014;14:102-114.

25. Tanabe M, Shimazu M, Wakabayashi G, Hoshino K, Kawachi S, Kadomura T, et al. Intraportal infusion therapy as a novel approach to adult ABO-incompatible liver transplantation. Transplantation 2002;73:1959-1961.

26. Tanabe M, Kawachi S, Obara H, Shinoda M, Hibi T, Kitagawa Y, et al. Current progress in $\mathrm{ABO}$-incompatible liver transplantation. Eur J Clin Invest 2010;40:943-949.

27. Takaya S, Iwaki Y, Starzl TE. Liver transplantation in positive cytotoxic crossmatch cases using FK506, high-dose steroids, and prostaglandin E1. Transplantation 1992;54:927-929.

28. Kozaki K, Egawa H, Kasahara M, Oike F, Yoshizawa A, Fukatsu A, et al. Therapeutic strategy and the role of apheresis therapy for $A B O$ incompatible living donor liver transplantation. Ther Apher Dial 2005;9:285-291.

29. Egawa H, Ohmori K, Haga H, Tsuji H, Yurugi K, Miyagawa-Hayashino $A$, et al. B-cell surface marker analysis for improvement of rituximab prophylaxis in $\mathrm{ABO}$-incompatible adult living donor liver transplantation. Liver Transpl 2007;13:579-588.

30. Uchiyama H, Mano Y, Taketomi A, Soejima Y, Yoshizumi T, Ikegami T, et al. Kinetics of anti-blood type isoagglutinin titers and $B$ lymphocytes in $\mathrm{ABO}$-incompatible living donor liver transplantation with rituximab and plasma exchange. Transplantation 2011;92:1134-1139.

31. Genberg H, Hansson A, Wernerson A, Wennberg L, Tydén G. Pharmacodynamics of rituximab in kidney allotransplantation. Am J Transplant 2006;6:2418-2428.

32. Skogsberg U, Breimer ME, Friman S, Mjörnstedt L, Mölne J, Olausson $M$, et al. Adult $A B O$-incompatible liver transplantation, using $A$ and B donors. Xenotransplantation 2006;13:154-159.

33. Kim JM, Kwon CHD, Joh JW, Han S, Yoo J, Kim K, et al. ABOincompatible living donor liver transplantation with rituximab and total plasma exchange does not increase hepatocellular carcinoma recurrence. Transplantation 2018;102:1695-1701.

34. Yoon YI, Song GW, Lee SG, Hwang S, Kim KH, Kim SH, et al. Outcome of $\mathrm{ABO}$-incompatible adult living-donor liver transplantation for patients with hepatocellular carcinoma. J Hepatol 2018;68:11531162. 
35. Pratschke J, Tullius SG. Promising recent data on ABO incompatible liver transplantation: restrictions may apply. Transpl Int 2007;20:647-648.
36. Toso C, Al-Qahtani M, Alsaif FA, Bigam DL, Meeberg GA, James Shapiro $A M$, et al. ABO-incompatible liver transplantation for critically ill adult patients. Transpl Int 2007;20:675-681. 